\title{
An index for evaluating distance of a healthy heart from Sino-Atrial blocking arrhythmia
}

\author{
${ }^{1}$ Electronic \& Robotic Engineering, Shahrood University Technology, Shahrood, Iran; \\ ${ }^{2}$ Ferdowsi University of Mashhad, Mashhad, Iran; \\ ${ }^{3}$ College of Engineering, Penn State University, Philadelphia, USA; \\ ${ }^{4}$ Sharif University of Technology, Tehran, Iran. \\ Email: Gholizade@shahroodut.ac.ir
}

Hossein Gholizade-Narm ${ }^{1}$, Morteza Khademi ${ }^{2}$, Asad Azemi ${ }^{3}$, Masoud Karimi-Ghartemani ${ }^{4}$

Received 29 November 2009; revised 15 December 2009; accepted 28 December 2009.

\begin{abstract}
In this paper, an index for evaluating Distance of a healthy heart from Sino-Atrial Blocking Arrhythmia (SABA) is presented. After definition of the main pacemakers' model of heart, Sino-Atrial (SA) and Atrio-Ventricular nodes (AV), the boundary of synchronization, which demonstrates the boundary of blocking arrhythmia, is obtained using perturbation method. In order to estimate of healthy heart characteristics, a parameter estimator is introduced. The distance from SABA is calculated using Lagrange method and Kohn-Tucker conditions. In addition, the maximum admissible decrease in the coupling intensity and the maximum admissible increase in the discrepancy between the natural frequencies of two pacemakers are determined in order to maintain the synchronization between the two pacemakers.
\end{abstract}

Keywords: Healthy Heart; Blocking Arrhythmia; Perturbation method; Synchronization Boundary; Optimization; Bifurcation

\section{INTRODUCTION}

The heart arrhythmias are the first cause of death according to World Health Organization (WHO) reports [1]. The lack of information about the health degree is one of the many factors for this. In other words, if people know their relative degree of health, the demise will be decreased. Fortunately, the medical science has the ability to measure the distance from illness in many issues. For instance, the fat and glucose of blood can be determined by some easy checks. The treatment could be initiated if it was near dangerous zone. Many works have also been done on cardiac arrhythmias' prediction to reduce the cardiac sudden death. Most of them have been done by physicians. The statistical methods are often used in those researches. In statistical methods, the
QRS complex and QT interval and other segments of Electro Cardio Graph (ECG) are investigated and cardiac sudden death is predicted $[2,3,4,5]$. The nonlinear features such as fractal dimensions of Heart Rate Variability (HRV) are used by other methods to determine the risk of sudden death [6]. Finally, the General Regression Neural Network (GRNN), Learning Vector Quantization (LVQ) and wavelet transform are used to predict the life-threatening Ventricular tachycardia (VT) and Ventricular Fibrillation (VF) [7].

All of the works are based on signal processing and their result is a number which demonstrates the probability of being prone to arrhythmia or death. Unfortunately, signal processing based methods are not of enough accuracy to predict life-threatening cardiac arrhythmias (less than 70\%) [6,7]. On the other hand, it cannot evaluate the relative degree of health if the heart is assumed healthy.

In this research, our goal is to evaluate the distance from SABA by observing action potential signals of SA and AV nodes. The blocking arrhythmias arise when two pacemakers are not in synchrony. The main reasons for asynchrony are either decreasing coupling intensity or increasing the discrepancy of two pacemakers' natural frequencies or both. For evaluation of distance from SABA, four stages should be passed. At first, a proper model should be considered for pacemakers. Then, the synchronization boundary must be obtained based on the model parameters. At the next stage, the model of parameters should be estimated from pacemakers' action potentials. Finally, by defining a cost function for distance from SABA and using optimization methods, the minimum distance is calculated.

Many models have been proposed for pacemakers and heart cells till now. Among the most well-known of them are Van der Pol [8,9,10,11], Fitzhugh-Nagumo [12,13,14], Hodgkin-Huxley [15], Beeler-Reuter [16] and Lou-Rudy [17] model. In this research, the Van der 
Pol model is used as the model of SA and AV nodes for its simplicity and sufficiency for synchronization issues.

Synchronization and its boundary have dedicated many works of engineers and physicists to itself from the past decades. Synchronization can be divided into complete synchronization, lag synchronization, functional synchronization, phase synchronization and frequency synchronization $[18,19]$. The output signal of the oscillators must be exactly equal for complete synchronization. In other words, two signals should be identical in amplitude and frequency. The lag synchronization is similar to complete synchronization except that the output of one oscillator should be coincided on the delayed signal of another one. The complete and lag synchronization are accessible only for two identical oscillators. In functional synchronization, the output signal of one oscillator is a function of another one. This type of synchronization is defined for those oscillators whose parameters are related together. Any type of the aforementioned synchronization types will not be occurred if two oscillators are non-identical. However, most of the coupled oscillators are non-identical in nature. In this case, the phase and frequency synchronization is defined in which, the coincidence of the output signals of two oscillators is not necessary. In phase synchronization, the phase difference of signals should be fixed for all the time and for frequency synchronization, it is enough that the phase difference of two oscillators to be bounded. The heart pacemakers are in phase synchrony in normal mode. When they tend to asynchrony, they pass frequency synchronization at first and then become asynchrony. Therefore, the boundary of synchronization is determined by frequency synchronization, but since it is difficult to find the frequency synchronization boundary (it can only be obtained by simulation [20]) and on the other hand, its distance from phase synchronization boundary if very tiny $[20,21]$, the phase synchronization has been taken as arrhythmia boundary in this research.

Perturbation methods, specially averaging method and bi-variant expansion are more applied in synchronization boundary determination $[20,21,22]$. The dynamical equations of two coupled oscillators are mapped to polar space using perturbation method. In polar space, the phase difference of two oscillators is a state variable. Therefore, the stability of new dynamical system is sufficient for phase synchronization because, in this case, the phase difference tends to a fixed point.

Unfortunately, there is not any background about measuring distance from arrhythmia. Our goal in this research is to evaluate this distance using synchronization boundary and pacemakers' parameters. In addition, we will answer to two questions: 1) How much the coupling intensity can be decreased subject to synchronization? 2) How much the natural frequency difference of two pacemakers can be increased subject to synchronization?

The paper is organized as follow: After introduction in Section 1, the synchronization boundary is obtained using perturbation method in Section 2. A state and parameter estimator is introduced in Section 3 for two coupled pacemakers system. The distance from SABA is evaluated in Section 4 and the maximum permitted value of natural frequency difference and minimum permitted value of coupling intensity are calculated in Section 5. Simulation results are demonstrated in Section 6 and the conclusion is discussed in Section 7.

\section{THE PACEMAKER MODEL AND SYNCHRONIZATION BOUNDARY}

The asynchrony between two vital pacemakers, SA and AV nodes, is the basic reason of blocking arrhythmias. In a healthy heart, the relationship between these two pacemakers is unidirectional coupling (from SA node to AV node) according to physicians' opinion; however, some physiologists suggest that the relationship is bidirectional but the effect of AV node on SA node is very smaller than vice versa [23]. According to the feedback of automatic nerves, the second idea is more acceptable. Here, we take the coupling as bidirectional, which is the general form. To change bidirectional case to unidirectional case, it is sufficient to equate the correspondence coefficient to the effect of AV node on SA node to zero. The model of two coupled pacemakers using Van der Pol Equations are,

$$
\begin{aligned}
& \ddot{x}_{t}-\mu_{1}\left(1-x_{t}^{2}\right) \dot{x}_{t}+\omega_{1}^{2} x_{t}=\alpha_{1}^{\prime}\left(y_{t}-x_{t}\right) \\
& \ddot{y}_{t}-\mu_{2}\left(1-y_{t}^{2}\right) \dot{y}_{t}+\omega_{2}^{2} y_{t}=\alpha_{2}^{\prime}\left(x_{t}-y_{t}\right)
\end{aligned}
$$

were $\mu_{1}$ and $\mu_{2}$ are damping coefficients, $\omega_{1}$ and $\omega_{2}$ are natural frequencies, and $\alpha_{1}^{\prime}$ and $\alpha_{2}^{\prime}$ are coupling coefficients which demonstrate coupling intensity. Assume Eqs. 1 and 2 are the SA and AV nodes' model respectively. According to relation between two coupled pacemakers, we take $\alpha_{1}^{\prime}=\delta \alpha_{2}^{\prime}, \delta<<1$. For using perturbation method, the system should be transformed to perturbation standard form. By scaling the time as $t=\varepsilon t_{1} \quad$ which results $\quad \dot{x}=\frac{d x}{d t}=\frac{d x}{d t_{1}} \frac{d t_{1}}{d t}=\frac{1}{\varepsilon} \dot{x}_{t_{1}}$, $\ddot{x}=\frac{1}{\varepsilon^{2}} \ddot{x}_{t_{1}}$ the Eqs. 1 and 2 change as,

$$
\begin{aligned}
& \ddot{x}_{t_{1}}-\varepsilon \mu_{1}\left(1-x_{t_{1}}^{2}\right) \dot{x}+x_{t_{1}}=\varepsilon \delta \alpha_{2}\left(y_{t_{1}}-x_{t_{1}}\right) \\
& \ddot{y}_{t_{1}}-\varepsilon \mu_{2}\left(1-y_{t_{1}}^{2}\right) \dot{y}_{t_{1}}+(1+\varepsilon \Delta) y_{t_{1}}=\varepsilon \alpha_{2}\left(x_{t_{1}}-y_{t_{1}}\right)
\end{aligned}
$$


where $\varepsilon^{2} \omega_{1}^{2}=1, \varepsilon^{2} \omega_{2}^{2}=1+\varepsilon \Delta$ and $\alpha_{2}=\varepsilon \alpha_{2}^{\prime}$. Suppose $\omega_{1} \gg 1$ therefore $\varepsilon<<1$. On the other hand, $\delta=k \varepsilon<<1$.

Remark: the natural frequency of SA node is greater than AV node in the healthy heart. Since our goal is the measurement of the distance of healthy heart from SABA, therefore $\Delta<0$ according to 3 .

Consider variables $x$ and $y$ linear versus $\varepsilon$ as $x=x_{0}+\varepsilon x_{1}, y=y_{0}+\varepsilon y_{1}$. By bi-variant expanding of Eq. 3 versus time in the form of $\xi=t_{1}, \eta=\varepsilon t_{1}$ and eliminating higher order terms $\left(O\left(\varepsilon^{2}\right)\right.$ and higher terms) we have,

$$
\begin{gathered}
\frac{\partial^{2} x_{0}}{\partial \xi^{2}}+x_{0}=0 \\
\frac{\partial^{2} x_{1}}{\partial \xi^{2}}+x_{1}=-2 \frac{\partial^{2} x_{0}}{\partial \xi \partial \eta}+\mu_{1}\left(1-x_{0}^{2}\right) \frac{\partial x_{0}}{\partial \xi} \\
\frac{\partial^{2} y_{0}}{\partial \xi^{2}}+y_{0}=0 \\
\frac{\partial^{2} y_{1}}{\partial \xi^{2}}+x_{1}=-2 \frac{\partial^{2} y_{0}}{\partial \xi \partial \eta}+\mu_{2}\left(1-y_{0}^{2}\right) \frac{\partial y_{0}}{\partial \xi} \\
+\alpha_{2}\left(\frac{\partial x_{0}}{\partial \xi}-\frac{\partial y_{0}}{\partial \xi}\right)-\Delta y_{0}
\end{gathered}
$$

Considering $x_{0}$ and $y_{0}$ in periodic forms,

$$
\begin{aligned}
& x_{0}=A(\eta) \cos \xi+B(\eta) \sin \xi \\
& y_{0}=C(\eta) \cos \xi+D(\eta) \sin \xi
\end{aligned}
$$

By substituting $x_{0}$ and $y_{0}$ at the right hand of Eqs. 4 and 6 and equating secular terms (coefficients of $\sin \xi$ and $\cos \xi$ ) to zero we have,

$$
\begin{gathered}
2 \frac{d A}{d \eta}=\mu_{1} A-\frac{1}{4} \mu_{1} A\left(A^{2}+B^{2}\right) \\
2 \frac{d B}{d \eta}=\mu_{1} B-\frac{1}{4} \mu_{1} B\left(A^{2}+B^{2}\right) \\
2 \frac{d C}{d \eta}=\mu_{2} C-\frac{1}{4} \mu_{2} C\left(C^{2}+D^{2}\right)+\alpha_{2}(D-B)+\Delta D \\
2 \frac{d D}{d \eta}=\mu_{2} D-\frac{1}{4} \mu_{2} D\left(C^{2}+D^{2}\right)+\alpha_{2}(A-C)+\Delta C
\end{gathered}
$$

Define A, B, C and D as follow,

$$
\begin{aligned}
& A=R_{1}(\eta) \cos \theta_{1}(\eta), B=R_{1}(\eta) \sin \theta_{1}(\eta) \\
& C=R_{2}(\eta) \cos \theta_{2}(\eta), D=R_{2}(\eta) \sin \theta_{2}(\eta)
\end{aligned}
$$

Therefore, $x_{0}$ and $y_{0}$ are,

$$
\begin{aligned}
& x_{0}(\xi, \eta)=R_{1}(\eta) \cos \left(\xi-\theta_{1}(\eta)\right) \\
& y_{0}(\xi, \eta)=R_{2}(\eta) \cos \left(\xi-\theta_{2}(\eta)\right)
\end{aligned}
$$

By substituting A, B, C and D in 9 and 10 and rearranging them,

$$
\begin{aligned}
& 2 \dot{R}_{1}=\mu_{1} R_{1}\left(1-\frac{1}{4} R_{1}^{2}\right) \\
& 2 R_{1} \dot{\theta}_{1}=0 \\
& 2 \dot{R}_{2}=\mu_{2} R_{2}\left(1-\frac{1}{4} R_{2}^{2}\right)-\alpha_{2} R_{1} \sin \left(\theta_{1}-\theta_{2}\right) \\
& 2 R_{2} \dot{\theta}_{2}=-\Delta-\alpha_{2}+\alpha_{2} R_{1} \cos \left(\theta_{1}-\theta_{2}\right)
\end{aligned}
$$

where $\dot{R}_{1}=\frac{d R_{1}}{d \eta}, \dot{R}_{2}=\frac{d R_{2}}{d \eta}, \dot{\theta}_{1}=\frac{d \theta_{1}}{d \eta}, \dot{\theta}_{2}=\frac{d \theta_{2}}{d \eta}$. Assume $\Phi=\theta_{1}-\theta_{2}$, the slow flow dynamical Equations are,

$$
\begin{aligned}
& 2 \dot{R}_{1}=\mu_{1} R_{1}\left(1-\frac{1}{4} R_{1}^{2}\right) \\
& 2 \dot{R}_{2}=\mu_{2} R_{2}\left(1-\frac{1}{4} R_{2}^{2}\right)-\alpha_{2} R_{1} \sin \Phi
\end{aligned}
$$

$$
2 \dot{\Phi}=\Delta+\alpha_{2}-\left(\alpha_{2} \frac{R_{1}}{R_{2}}\right) \cos \Phi
$$

where $\dot{\Phi}=\frac{d\left(\theta_{1}-\theta_{2}\right)}{d \eta}$. The synchronization boundary is achieved by determining the Saddle-Node (S-N) and Hopf bifurcation condition curves of 14 [20]. The first step is to find the equilibrium points curve. Equate the left hand of 14 to 0 ,

$$
\begin{aligned}
& \mu_{1} R_{1}\left(1-\frac{1}{4} R_{1}^{2}\right)=0 \Rightarrow R_{1}=2 \\
& \mu_{2} R_{2}\left(1-\frac{1}{4} R_{2}^{2}\right)-\alpha_{2} R_{1} \sin \Phi=0 \\
& \Rightarrow \sin \Phi=\frac{\mu_{2} R_{2}\left(1-\frac{1}{4} R_{2}^{2}\right)}{\alpha_{2} R_{1}}
\end{aligned}
$$




$$
\begin{aligned}
& \Delta+\alpha_{2}-\left(\alpha_{2} \frac{R_{1}}{R_{2}}\right) \cos \Phi=0 \\
& \Rightarrow \cos \Phi=\frac{\left(\Delta+\alpha_{2}\right) R_{2}}{\alpha_{2} R_{1}}
\end{aligned}
$$

By summing the square of 16 and 17 and substituting $\mathrm{R}_{1}=2$ we have,

$$
\left(\mu_{2} R_{2}\left(1-\frac{1}{4} R_{2}^{2}\right)\right)^{2}+\left(\left(\Delta+\alpha_{2}\right) R_{2}\right)^{2}=4 \alpha_{2}^{2}
$$

Replace $R_{2}^{2}=p$ and rearrange 18 according to power of $p$,

$$
\frac{1}{16} \mu_{2}^{2} p^{3}-\frac{1}{2} \mu_{2}^{2} p^{2}+\left(\left(\Delta+\alpha_{2}\right)^{2}+\mu_{2}^{2}\right) p-4 \alpha_{2}^{2}=0(
$$

Eq. 19 shows the curve of equilibrium points vs. the system's parameters. Using Descartes' rule of signs we see that (19) has either one or three positive roots for $p$. At bifurcation, there will be a double root which corresponds to requiring the derivative of (19) to vanish:

$$
\frac{3}{16} \mu_{2}^{2} p^{2}+\mu_{2}^{2} p+\left(\left(\Delta+\alpha_{2}\right)^{2}+\mu_{2}^{2}\right)=0
$$

Eliminating $p$ from (19) and (20) gives the condition for S-N bifurcation as,

$$
\begin{aligned}
& 4 \Delta^{6}+24 \Delta^{5} \alpha_{2}+\left(60 \alpha_{2}^{2}+8 \mu_{2}^{2}\right) \Delta^{4}+\left(32 \mu_{2}^{2} \alpha_{2}+80 \alpha_{2}^{3}\right) \Delta^{3} \\
& +\left(60 \alpha_{2}^{4}+4 \mu_{2}^{4}+12 \mu_{2}^{2} \alpha_{2}^{2}\right) \Delta^{2} \\
& +\left(8 \mu_{2}^{4} \alpha_{2}+24 \alpha_{2}^{5}-40 \mu_{2}^{2} \alpha_{2}^{3}\right) \Delta+4 \alpha_{2}^{6}-\mu_{2}^{2} \alpha_{2}^{4}=0
\end{aligned}
$$

Eq. 21 plots as two curves intersecting at cusp points in the $\left(\alpha_{2}, \Delta\right)$ plane. At the cusp point, a further degeneracy occurs and there is a triple root in Eq. 19. Derivation from 20 gives,

$$
p=\frac{8}{3}
$$

By substituting $p$ from (22) to (19) and (20) gives cusp points.

$$
\begin{aligned}
& \left(\alpha_{2}^{*}=0.544 \mu_{2}, \Delta_{1}^{*}=0.033 \mu_{2}\right), \\
& \left(\alpha_{2}^{*}=0.544 \mu_{2}, \Delta_{2}^{*}=-1.122 \mu_{2}\right)
\end{aligned}
$$

Next we look for Hopf bifurcations in the slow flow system 14. Let $R_{10}, R_{20}, \Phi_{0}$ be an equilibrium point. The behavior of the system linearized in the neighborhood of this point is determined by the eigenvalues of the Jacobian matrix.

$$
J=\frac{1}{2}\left[\begin{array}{ccc}
-\frac{3 R_{10}^{2}-4}{4} \mu_{1} & 0 & 0 \\
-\alpha_{2} \sin \left(\Phi_{0}\right) & -\frac{3 R_{20}^{2}-4}{4} \mu_{2} & -R_{10} \alpha_{2} \cos \left(\Phi_{0}\right) \\
-\frac{\alpha_{2}}{R_{20}} \cos \left(\Phi_{0}\right) & \frac{\alpha_{2} R_{10}}{R_{20}^{2}} \cos \left(\Phi_{0}\right) & \frac{\alpha_{2} R_{10}}{R_{20}} \sin \left(\Phi_{0}\right)
\end{array}\right]
$$

Substituting $\sin \left(\Phi_{0}\right), \cos \left(\Phi_{0}\right), R_{10}$ from (15-17) in 24 and after simplification,

$$
J=\frac{1}{2}\left[\begin{array}{ccc}
-2 \mu_{1} & 0 & 0 \\
-\frac{1}{2} \mu_{2} R_{2}\left(1-\frac{1}{4} R_{2}^{2}\right) & \left(1-\frac{3}{4} R_{20}^{2}\right) \mu_{2} & -\left(\Delta+\alpha_{2}\right) R_{20} \\
-\frac{\left(\Delta+\alpha_{2}\right)}{2} & \frac{\left(\Delta+\alpha_{2}\right)}{R_{20}} & \mu_{2}\left(1-\frac{1}{4} R_{20}^{2}\right)
\end{array}\right]
$$

For Hopf bifurcation, it is necessary that matrix $J$ contains 2 pure imaginary and 1 real eigenvalue.

$$
\begin{aligned}
& |\lambda I-J|=0 \Rightarrow \\
& \left(\lambda+\mu_{1}\right)\left(\lambda^{2}+\left(\frac{1}{2} \mu_{2} R_{20}^{2}-\mu_{2}\right) \lambda+J_{22} J_{33}-J_{23} J_{32}\right)=0
\end{aligned}
$$

It can be seen from 26 that the coefficient of $\lambda$ must be 0 for Hopf bifurcation.

$$
\frac{1}{2} \mu_{2} R_{20}^{2}-\mu_{2}=0 \Rightarrow p=R_{20}^{2}=2
$$

Substituting p from 27 in Eq. 19 gives the Hopf bifurcation condition curve.

$$
\Delta^{2}+2 \alpha_{2} \Delta+\frac{1}{4} \mu_{2}^{2}-\alpha_{2}^{2}=0
$$

Eqs. 21 and 28 identify the phase synchronization boundary. A typical sample for synchronization boundary is brought in Ex. 1.

Synchronization boundary Equations show that the boundary depends on coupling coefficients, damping coefficients and discrepancy of two pacemakers' natural frequency. Therefore, they should be estimated in practice for distance evaluation from SABA.

\section{THE STATE-PARAMETER ESTIMATOR}

Measurement of all states and parameters of the cardiac pacemakers is impossible in practice but the measurement of only their action potentials is practical using two implantable leads. In this section, a state-parameter estimator (adaptive observer) is introduced which is able to 
estimate necessary parameters from pacemakers' action potentials for distance evaluation. This estimator uses the high-gain method. The high-gain method is very effective in state estimation [24]. When the parameters are unknown in addition to states, the estimator is modified which is called adaptive observer. An adaptive observer is introduced in [25] which can estimate parameters and states simultaneously. We use an adaptive observer which is introduced in [25] in this research. The system form is as follow,

$$
\begin{aligned}
& \dot{x}(t)=\left(\begin{array}{ll}
A_{1} & 0 \\
0 & A_{1}
\end{array}\right) x(t)+\left(\begin{array}{l}
\phi_{1}(x(t), u(t)) \\
\phi_{2}(x(t), u(t))
\end{array}\right) \\
& +\left(\begin{array}{l}
\psi_{1}(x(t), u(t)) \\
\psi_{2}(x(t), u(t))
\end{array}\right) \theta \\
& y(t)=\left(\begin{array}{ll}
C_{1} & 0 \\
0 & C_{2}
\end{array}\right) x(t)
\end{aligned}
$$

where $x \in \mathfrak{R}^{n}, \quad y \in \mathfrak{R}^{2}$, and $\theta \in \mathfrak{R}^{q}$ are the state vector, output vector and the unknown parameters vector respectively. The matrices $C_{i}, \psi_{i}, A_{i}, \mathrm{i}=1,2$ are,

$$
\begin{aligned}
& A_{i}=\left[\begin{array}{cccc}
0 & 1 & & 0 \\
& & & \\
& & \ddots & \\
& & & 1 \\
& & & \\
0 & & & 0
\end{array}\right], \\
& \psi_{i}(x, u)=\left[\begin{array}{ccc}
0 & \cdots & 0 \\
\vdots & & \vdots \\
0 & \cdots & 0 \\
\psi_{i n_{i}, 1}(x, u) & \cdots & \psi_{i n_{i}, q}(x, u)
\end{array}\right] \\
& C_{i}=\left[\begin{array}{llll}
1 & 0 & \cdots & 0
\end{array}\right]_{1 \times n_{i}}, n=\sum_{i=1}^{2} n_{i}
\end{aligned}
$$

The adaptive rules are,

$$
\begin{aligned}
& \dot{\hat{\Gamma}}(t)=\lambda(A-K C) \hat{\Gamma}(t)+\lambda \psi(\tilde{x}(t), u(t)) \\
& \dot{\hat{x}}(t)=A \hat{x}(t)+\phi(\tilde{x}(t), u(t))+\psi(\tilde{x}(t), u(t)) \tilde{\theta}(t) \\
& +\Lambda(\lambda)^{-1}\left[\lambda K+\Gamma(t) \Gamma^{T}(t) C^{T}\right][y(t)-C \tilde{x}(t)] \\
& \dot{\hat{\theta}}(t)=I_{\lambda} \Gamma^{T}(t) C^{T}[y(t)-C \tilde{x}(t)] \\
& \tilde{x}=\hat{x} \text { if }\|\hat{x}\| \leq X, \frac{\hat{x}}{\|\hat{x}\|} X \text { otherwise. } \\
& \tilde{\theta}=\hat{\theta} \text { if }\|\hat{\theta}\| \leq \Theta, \frac{\hat{\theta}}{\|\hat{\theta}\|} \text { otherwise. }
\end{aligned}
$$

where $\Theta, X$ are the $\theta(0), x(0)$ upper bound,

$$
K=\left[\begin{array}{cc}
K_{1} & 0 \\
0 & K_{2}
\end{array}\right]
$$

and $K_{1,2}$ are selected such that the $A_{i}-K_{i} C_{i}$ matrix be stable.

$$
\begin{gathered}
\Lambda^{-1}{ }_{i=1,2}(\lambda)=\operatorname{diag}\left(1, \lambda, \lambda^{2}, \cdots, \lambda^{n_{i}-1}\right)(\lambda>0), \\
\Lambda=\left[\begin{array}{ll}
\Lambda_{1} & 0 \\
0 & \Lambda_{2}
\end{array}\right] \\
I_{\lambda}=\left[\begin{array}{cc}
\lambda^{n_{1}} I & 0 \\
0 & \lambda^{n_{2}} I
\end{array}\right]
\end{gathered}
$$

For using the introduced adaptive observer, the system 1 and 2 must be mapped to state space representation.

$$
\begin{aligned}
& \dot{x}(t)=A x(t)+\phi(x(t), u(t))+\psi(x(t), u(t)) \theta \\
& y(t)=C x(t)
\end{aligned}
$$

where, 


$$
\begin{aligned}
& C=\left[\begin{array}{cccc}
1 & 0 & 0 & 0 \\
0 & 0 & 1 & 0
\end{array}\right], A=\left[\begin{array}{cccc}
0 & 1 & 0 & 0 \\
0 & 0 & 0 & 0 \\
0 & 0 & 0 & 1 \\
0 & 0 & 0 & 0
\end{array}\right], x(t)=\left[\begin{array}{llll}
x_{1}(t) & x_{2}(t) & x_{3}(t) & x_{4}(t)
\end{array}\right]^{T} \\
& \theta=\left[\begin{array}{llllll}
\mu_{1} & \omega_{1}^{2} & \alpha_{1}^{\prime} & \mu_{2} & \omega_{2}^{2} & \alpha_{2}^{\prime}
\end{array}\right]^{T}, \phi(x, u)=0 \\
& \psi(x, u)=\left[\begin{array}{cccccc}
0 & 0 & 0 & 0 & 0 & 0 \\
\left(1-x_{1}^{2}(t)\right) x_{2}(t) & -x_{1}(t) & -x_{1}(t)+x_{3}(t) & 0 & 0 & 0 \\
0 & 0 & 0 & 0 & 0 & 0 \\
0 & 0 & 0 & \left(1-x_{3}^{2}(t)\right) x_{4}(t) & -x_{3}(t) & x_{1}(t)-x_{3}(t)
\end{array}\right]
\end{aligned}
$$

The performance of introduced estimator is demonstrated in Ex. 2.

\section{DISTANCE EVALUATION FROM SABA}

In this section, our goal is to evaluate the distance from SABA using synchronization boundary and pacemakers' parameters. As it was mentioned in Section 2, the boundary is determined by S-N and Hopf bifurcation conditions. If $-1.122 \mu_{2} \leq \Delta<0$, the boundary is determined by $\mathrm{S}-\mathrm{N}$ bifurcation condition curve. Otherwise, the synchronization boundary is identified by Hopf bifurcation condition curve. For distance evaluation, at first, the pacemakers' parameters should be mapped from $\left(\alpha^{\prime}, \mu, \omega_{1}, \omega_{2}\right)$ space to $(\alpha, \mu, \Delta)$ space.

$$
\varepsilon=\frac{1}{\omega_{1}}, \Delta=\varepsilon\left(\omega_{2}^{2}-\omega_{1}^{2}\right), \alpha_{2}=\varepsilon \alpha_{2}^{\prime}
$$

The second step is to find a point on synchronization boundary in which has the minimum distance from $(\alpha, \mu, \Delta)$. Assume the point $\left(\alpha_{2}^{*}, \mu_{2}^{*}, \Delta^{*}\right)$ is the desired point. By inverse transformation of this point using (35), the minimum distance from SABA can be obtained.

$$
\omega_{2 \min }^{2}=\omega_{1}^{2}+\frac{1}{\varepsilon} \Delta^{*}, \alpha_{2}^{\prime *}=\frac{1}{\varepsilon} \alpha_{2}^{*}
$$

Min. Dist. from Blocking Arrh. =

$$
\sqrt{\left(\omega_{2}-\omega_{2 \min }\right)^{2}+\left(\alpha_{2}^{\prime}-\alpha_{2}^{\prime *}\right)^{2}+\left(\mu_{2}-\mu_{2}^{*}\right)^{2}}
$$

The crucial point is how to find the desired point. The Eq. 21 contains two curves that one of them pass through origin point $(0,0,0)$ and forms the first part of synchronization boundary. For simplicity, we approximate this curve by a line which passes from origin and cusp point.

$$
\alpha_{2}=-0.48485 \Delta
$$

Therefore, the synchronization boundary is defined by Eqs. 28 and 37. Suppose $P=\left(\alpha_{2 P}, \mu_{2 P}, \Delta_{P}\right)$ is the pacemakers' parameters which is transformed to $(\alpha, \mu, \Delta)$ space. Define the distance of a point on boundary $Q=(\alpha, \mu, \Delta)$ from $\mathrm{P}$ as,

$$
\operatorname{Dis}^{2}=\left(\alpha_{2}-\alpha_{2 P}\right)^{2}+\left(\mu_{2}-\mu_{2 P}\right)^{2}+\left(\Delta-\Delta_{P}\right)^{2}
$$

By minimizing 38 subject to 37 and again subject to 28 individually and taking into account their minimum value, the desired point $\left(\alpha_{2}^{*}, \mu_{2}^{*}, \Delta^{*}\right)$ is achieved. Therefore, two minimization problems should be solved.

$$
\begin{aligned}
& \text { Minimize } \text { Dis }^{2} \\
& \text { S.T. } f_{1}\left(\alpha_{2}, \mu_{2}, \Delta\right)=\alpha_{2}+0.48485 \Delta=0 \\
& \quad-1.122 \mu_{2} \leq \Delta \leq 0
\end{aligned}
$$

Using Lagrange coefficient and Kohn-Tucker method for considering inequality constraints we have,

$$
\begin{gathered}
L_{1}=D i s^{2}-\lambda f_{1}\left(\alpha_{2}, \mu_{2}, \Delta\right)+v_{1} \Delta-v_{2}\left(\Delta+1.122 \mu_{2}\right)(40 \\
\nabla_{\left(\alpha_{2}, \mu_{2}, \Delta\right)} L_{1}=0 \\
\nabla_{\lambda} L_{1}=0 \\
v_{1} \Delta=0 \\
-v_{2}\left(\Delta+1.122 \mu_{2}\right)=0
\end{gathered}
$$


The parameters $\left(\alpha_{2}, \mu_{2}, \Delta, \lambda, v_{1}, v_{2}\right)$ are gained by solving 41. If conditions 42 and 43 are satisfied, the distance is evaluated from obtained $\left(\alpha_{2}, \mu_{2}, \Delta\right)$, otherwise, the extremum of those parameters which do not satisfy the conditions is substituted to evaluate distance.

$$
\begin{aligned}
& \Delta \leq 0 \\
& -\left(\Delta+1.122 \mu_{2}\right) \leq 0
\end{aligned}
$$

and

$$
\begin{aligned}
& v_{1} \geq 0 \\
& v_{2} \geq 0
\end{aligned}
$$

In second time, the desired point is sought on the (37) surface.

$$
\begin{aligned}
& \text { Minimize } \text { Dis }^{2} \\
& \text { S.T. } \quad f_{2}\left(\alpha_{2}, \mu_{2}, \Delta\right)=\Delta^{2}+2 \alpha_{2} \Delta+\frac{1}{4} \mu_{2}^{2}-\alpha_{2}^{2}=0 \text { (44) } \\
& \quad \Delta \leq-1.122 \mu_{2}
\end{aligned}
$$

Again, using Lagrange coefficient and Kohn-Tucker method, $\left(\alpha_{2}, \mu_{2}, \Delta\right)$ values are obtained and distance is evaluated. By comparing the two evaluated distance and considering the minimum value, the desired point is identified. By inverse transformation of this point to $\left(\alpha^{\prime}, \mu, \omega_{1}, \omega_{2}\right)$ space according to Eq. 35 , the minimum distance from SABA is evaluated using (36). The distance evaluation is illustrated in Ex. 3 for a typical sample.

\section{IDENTIFICATION OF MAXIMUM ADMISSIBLE VARIATION OF PACEMAKERS' PARAMETERS}

In this section, our goal is to evaluate the maximum variation of a parameter subject to remaining synchronization whiles other parameters are fixed. For instance, how much the coupling coefficient can be decreased whiles two pacemakers remain in phase synchrony or how much the discrepancy of two natural frequencies can be increased and the synchronization remain unchanged.

\subsection{Maximum Admissible Decrease of Coupling Coefficient}

For identification of maximum admissible variation of coupling coefficient, at first, we transform the pacemakers' parameters to same usable form in synchronization boundary equations using Eq. 34. Now, we consider coupling coefficient as unknown parameter and obtain it from Eq. 37. If the condition (42) is satisfied, the obtained value is the desired coefficient otherwise we will compute it from Eq. 28. In any way, assume the desired coefficient is $\alpha_{2}^{*}$. Therefore the maximum admissible decrease of coupling coefficient is,

$$
\delta_{\max } \alpha_{2}=\alpha_{2}^{\prime}-\frac{\alpha_{2}^{*}}{\varepsilon}
$$

In other words, the minimum of coupling coefficient subject to remaining synchronization is,

$$
\alpha_{2 \min }^{\prime}=\frac{\alpha_{2}^{*}}{\varepsilon}
$$

\subsection{Maximum Admissible Increase Discrepancy between Two Pacemakers' Natural Frequencies}

For identification of maximum admissible increase discrepancy between two Pacemakers' natural frequencies, like Section 5-1, at first, we transform the pacemakers' parameters from $\left(\alpha^{\prime}, \mu, \omega_{1}, \omega_{2}\right)$ space to $(\alpha, \mu, \Delta)$ space using Eq. 34. Now, we assume that $\Delta$ is the unknown parameter and obtain it from Eq. 37. If the condition (42) is satisfied, the obtained value is desired $\Delta$ otherwise we compute it from Eq. 28. In any way, assume the desired value is the $\Delta^{*}$. Therefore the maximum admissible increase of discrepancy between two Pacemakers' natural frequencies is,

$$
\begin{aligned}
& \delta_{\text {max }} \omega=\omega_{1}-\omega_{2 \min } \\
& \omega_{2 \text { min }}^{2}=\omega_{1}^{2}+\frac{1}{\varepsilon} \Delta^{*}
\end{aligned}
$$

The damping coefficient is one of the determinative parameters for synchronization boundary but we ignore to find its maximum admissible variation for its little importance.

\section{SIMULATION}

The distance evaluation from SABA is completed in 3 steps: at first, the synchronization boundary is determined, and then the pacemakers' parameters estimated. Finally, the minimum distance from boundary is computed. In this section, the synchronization boundary is obtained for a typical sample and plotted (Ex. 1). Afterward, the performance of introduced adaptive observer is illustrated in Ex. 2. Then the distance from SABA is shown in Ex. 3. As a final point, the maximum admissible variation of parameters is calculated in Ex. 4 .

Ex. 1. Assume $\mu_{2}=1$, the synchronization boundary is determined by (21) and (28). Figure 1 shows the synchronization boundary and highlights the cusp points.

Ex. 2. Assume pacemakers' parameters as $\mu_{1}=2$, $\omega_{1}^{2}=64, \alpha_{1}^{\prime}=4, \mu_{2}=1, \omega_{2}^{2}=25, \alpha_{2}^{\prime}=22$. The parameters are chosen such that the synchronization frequency is 70 per minute and the natural frequency of pacemakers equal to 76 and 47 per minute for SA and AV nodes respectively. Figure 2 illustrates the state- 


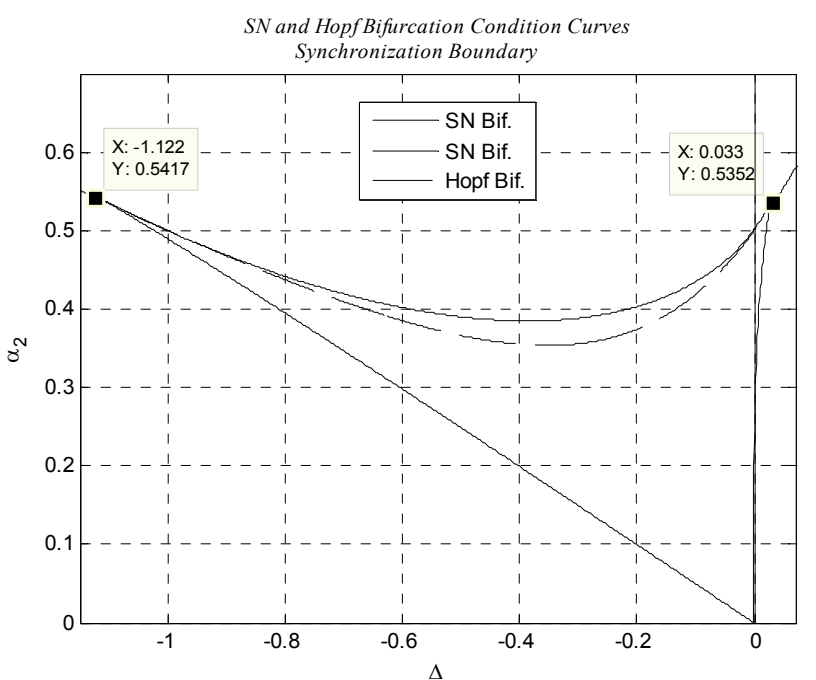

(a)

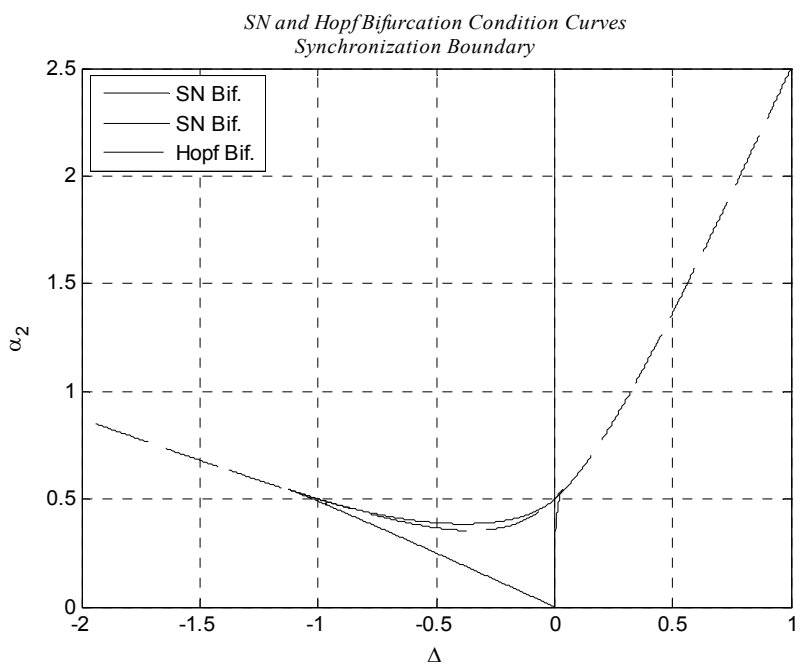

(b)

Figure 1. Phase synchronization Boundary. a. small discrepancy of natural frequency b. big discrepancy of natural frequency.

parameter estimator performance.

Ex. 3. Suppose pacemakers' parameters as Ex. 2 for using synchronization boundary to evaluate distance from SABA, we should transform parameters at first.

$$
\begin{aligned}
& \varepsilon=0.125, \delta=0.05, \\
& P=\left(\alpha_{2 P}=\varepsilon \alpha_{2}^{\prime}, \mu_{2 P}, \Delta_{P}\right)=(2.75,1,-4.875)
\end{aligned}
$$

Since $\Delta_{P}=-4.875<<-1.122$, therefore the desired point is on Hopf bifurcation condition curve. By solving (44) we have, $\alpha_{2}^{*}=2.1409, \mu_{2}^{*}=1.0214, \Delta^{*}=-5.1252$. We see that condition (44) is satisfied. The distance from SABA is,
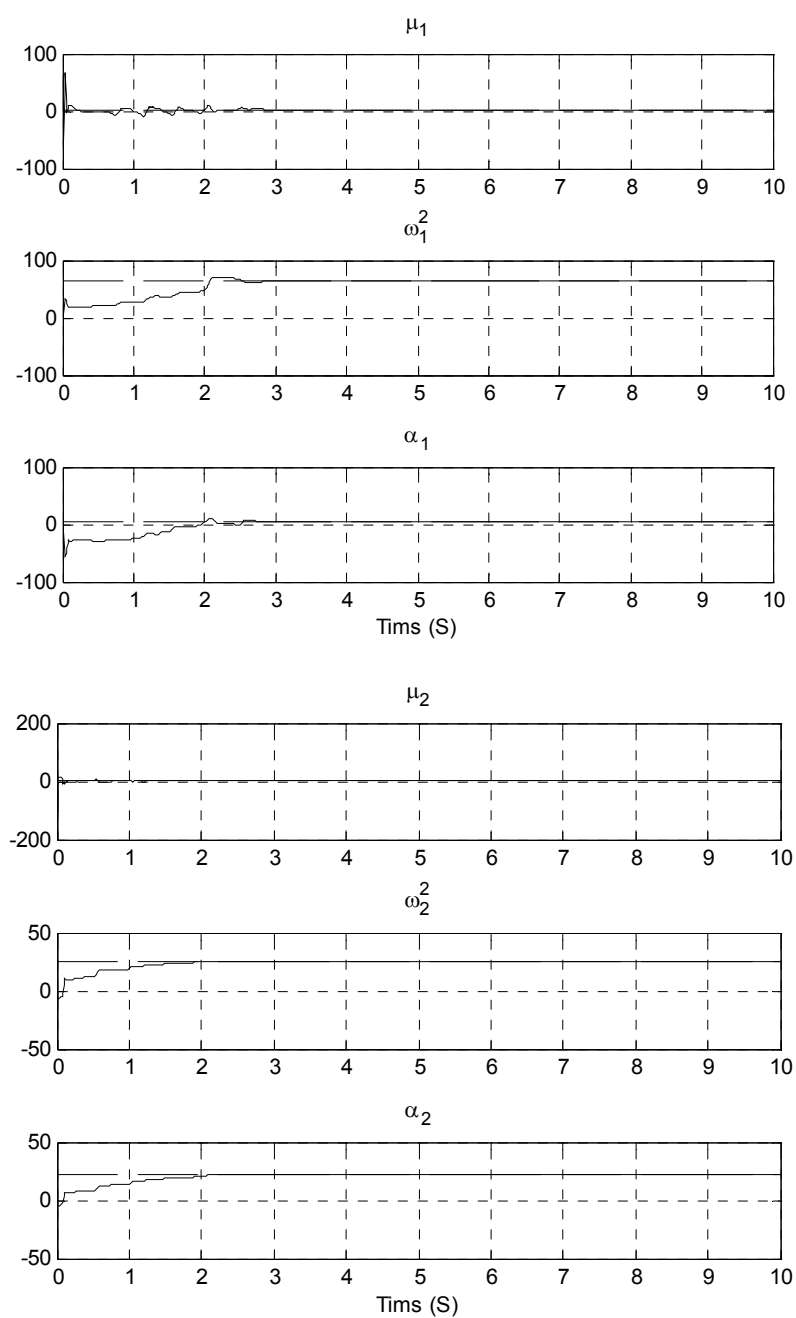

Figure 2. System's parameter estimation.

$\omega_{2 \min }^{2}=64-8 \times 5.1252=22.9284$,

$\alpha_{2}^{*}=8 \times 2.1409=17.1272, \quad \mu_{2}^{*}=1.0214$

Min. Dist. from Blocking Arrh.

$=\sqrt{\left(\omega_{2}-\omega_{2 \min }\right)^{2}+\left(\alpha_{2}^{\prime}-\alpha_{2}^{*}\right)^{2}+\left(\mu_{2}-\mu_{2}^{*}\right)^{2}}=23.7894$

Ex. 4. The maximum admissible variation for Ex. 3 parameters are,

$$
\begin{aligned}
& \alpha_{2}^{*}=2.0374 \Rightarrow \alpha_{2}^{\prime *}=\frac{1}{\varepsilon} \alpha_{2}^{*} \\
& =16.2992 \Rightarrow \delta_{\max } \alpha_{2}^{\prime}=5.7008 \\
& \Delta^{*}=-6.61 \Rightarrow \delta_{\max } \Delta=-1.7318, \omega_{2 \min }^{2} \\
& =11.1456 \Rightarrow \delta_{\max } \omega_{2}=\omega_{2}-\omega_{2 \min }=1.6615
\end{aligned}
$$

\section{CONCLUSIONS}

The evaluation of distance from SA blocking arrhythmia for a healthy heart was investigated in this paper. To do 
this, the synchronization boundary was obtained using perturbation method. The synchronization boundary defines the illness boundary approximately. To compute the distance from this boundary, an estimator was introduced which can estimate SA and AV nodes' parameters from their action potential signals. Using optimization methods, the minimum distance from SABA was evaluated. This value can be considered as an alarm when it is very small. The coupling intensity and discrepancy of natural frequency of two pacemakers play a crucial role in synchronization of two basic pacemakers. Hence, a method was proposed to evaluate the maximum admissible variation of these parameters. The maximum variation value demonstrates the confidence margin.

\section{REFERENCES}

[1] World Helth Organization (WHO), Internet Available: www.who.int

[2] El-Sherif, N., Denes, P., Katz, R., Capone, R., Brent , L., Carlson, M. and Reynolds, R. (1995) Definition of the best prediction criteria of the time domain signal-averaged electrocardiogram for serious arrhythmic events in the post infraction period. Journal of American Collection Cardiology, 25(4), 908-914.

[3] Tsagalou, E. P., Anastasiou-Nava, M. I., Karagounis, L. A., Alexopoulos, G. P., Batziou, C., Toumanidis, S., Papadaki, E. and Nanas, J. N. (2002) Dispersion of QT and QRS in patients with severe congestive heart failure: Relation to cardiac and sudden death mortality, Hellenic Jornal of Cardiology, 43, 209-215.

[4] Thong, T., McNames, J., Aboy, M. and Goldstein, B. (2003) Paroxysmal atrial fibrillation prediction using isolated premature atrial events and paroxysmal atrial tachycardia. Proceedings of IEEE International Conference on Biomedical Engineering, EMBC, 163-166.

[5] Thong, T., McNames, J., Aboy, M. and Goldstein, B. (2004) Prediction of paroxysmal atrial fibrillation by analysis of atrial premature complexes. IEEE Transactions On Biomedical Engineering, 51(4), 561-569.

[6] Owis, I., Abou-Zied, H. and Youssef, M. (2002) Study of Features Based on Nonlinear Dynamical Modeling in ECG Arrhythmia Detection and Classification. IEEE Transactions On Biomedical Engineering, 49(7), 733-736.

[7] Abbas, R., Aziz, W. and Arif, M. (2004) Prediction of ventricular Tachyarrhythmia in Electrocardiograph Signal Using Neuro-wavelet Approach. National Conference on Emerging Technologies, 82-87.

[8] Van der Pol, B. and Van der mark, J. (1927) Frequency demultiplication. Nature, 120, 363-364.

[9] Van der Pol, B. and Van der mark, J. (1928) The Heartbeat considered as a relaxation oscillation and electrical model of heart. Phil. Mag. Supll., 6, 763-775.
[10] Grudzinsky, K. and Zebrowski, J. (2004) Modeling cardiac pacemakers with relaxation oscillators. Physica A: Statistical Mechanics and its Applications, 153-162.

[11] Sato, S., Doi, S. and Nomura, T. (1994) Bonhoffer-van der pol oscillator model of the Sino-Atrial node: A possible mechanism of heart rate regulation. Method of Information in Medicin, 116-119.

[12] Fitzhugh, R. (1961) Impulses and physiological in theoretical models of nerve membranes. Biophysical Journal, 1, 445-466.

[13] FitzHugh, R. (1969) Mathematical models of excitation and propagation in nerve. In: Schwan H.P. Ed., Biological Engineering, McGraw Hill, New York.

[14] Nagumo, J., Arimoto, S. and Yoshizawa, S. (1962) An active pulse transmission line simulating nerve axon. Proceedings of the IRE, 50, 2061-2070.

[15] Hodgkin, A. L., Huxley, A. F. and Katz, B. (1952) Measurement of current-voltage relations in the membrane of the giant axon of Loligo, The Journal of physiology, 116, 424.

[16] Beeler, G.W. and Reuter, H. (1977) Reconstruction of the action potential of ventricular myocardial fibres. The Journal of physiology, 268, 177-210.

[17] Lou, C.H. and Rudy, Y. (1994) A dynamic model of the cardiac ventricular action potential I. Circulation Research, 74, 1071-1096.

[18] Pikovsky, A., Rosenblum, M. and Kurths, J. (2002) Synchronization: A universal concept nonlinear science, Cambridge University Press.

[19] Santos, A. M., Lopes, S. R. and Viana, R. L. (2004) Rhythm synchronization and chaotic modulation of couplead van der pol oscillatiors in a model for the heartbeat. Physica A, 338, 335-355.

[20] Rand R. H. (2004) Lecture notes in nonlinear vibrations. 45 version, The Internet-First University Press, Ithaca. http://dspace.library.cornell.edu/handle/1813/79.

[21] Rompala, K., Rand, R. and Howland, H. (2007) Dynamics of three coupled van der Pol oscillators with application to circadian rhythms. Communications in Nonlinear Science and Numerical Simulation, 12, 794-803.

[22] Nayfeh, A. H. (1973) Perturbation Methods, John Wiley \& Sons Ltd., Chichester.

[23] Grudzinski, K., Zebrowski, J.J. and Baranowski, R. (2006) Model of the sino-atrial and atrio-ventricular nodes of the conduction system of the human heart. Biomedical Technology, 51, 210-214.

[24] Gauthier, J. P., Hammouri, H. and Othman, S. (1992) A simple observer for nonlinear systems - applications to bioreactors. IEEE Transactions on Automatic Control. 37(6), 875-880.

[25] Besancon, G., Zhang, Q. and Hammouri, H., (2002) High-Gain observer based state and parameter estimation in nonlinear systems. International Federation of Automatic Control. 